\title{
Evolution récente du refroidissement du lait à la production (1)
}

\author{
par \\ André GAC (I.N.A. 1943) \\ Docteur Ingénieur - Ingénieur en chef du G.R.E.F. \\ Chef de la Division Industries Agricoles et Alimentaires \\ et Froid du Centre Technique d'Antony
}

En 1961 puis en 1964, l'Association Française du Froid avec l'Association Laitière Française organisaient à Auxerre et à La Rochelle des Journées Nationales d'Etudes sur le refroidissement du lait à la production. L'objectif de ces deux manifestations était, en particulier, d'informer les professionnels des possibilités, avantages et servitudes des différents procédés de refroidissement du lait. A cette époque, en effet, les techniques étaient variées et certaines nous paraissent aujourd'hui curieuses : fusion de glace de lait ; ruissellement du lait sur des surfaces refroidies ; emploi de " tourniquets " posés sur les bidons, etc.

En 1971, débutait la normalisation des refroidisseurs de lait, sur le plan européen dans le cadre des travaux de la Commission Européenne de Normalisation (CEN) et, sur le plan mondial, au Comité Technique 23 de l'ISO.

Les années 1964 et 1971 peuvent être considérées, en France, comme des jalons importants marquant le développement du refroissement de lait. En l'espace de 7 ans, cette technique s'est imposée, pour des motifs que ceux qui la préconisaient n'avaient probablement pas imaginés et dans des conditions pratiques qui, il y a 20 ans encore, paraissent inadmissibles.

\section{I. - LES CAUSES DE LA GENERALISATION DU REFROIDISSEMENT A LA PRODUCTION}

L'une des servitudes essentielles de la production laitière est qu'elle impose un certain travail quotidien. Cette servitude est de

(1) Cahiers des Ingénieurs agronomes, 1974, nº 282. 
plus en plus difficilement admise ; le personnel des usines laitières et les ramasseurs demandent "leur droit au dimanche » ; les producteurs entendent organiser leur travail à leur convenance et ne plus être tributaires de l'horaire de la collecte. Ces motifs, qui ne sont pas d'ordre économique ou technologique, ont cependant conduit à l'adoption généralisée du refroidissement intense du lait sitôt la traite, entre $0^{\circ} \mathrm{C}$ et $4^{\circ} \mathrm{C}$, de façon à faire passer le rythme de la collecte d'un ramassage journalier à un ramassage tous les 2 j. L'usine laitière peut ainsi fermer ses portes les dimanches et les jours fériés.

Mais si le ramassage s'effectue $1 \mathrm{j}$ sur 2 , il faut doubler la capacité de transport, c'est-à-dire, soit doubler le nombre de camions, soit transporter deux fois plus de lait; cette dernière solution a fait adopter la citerne dans laquelle sont mélangées toutes les productions. Cette pratique est contraire à la règle qui paraissait fondamentale du "non mélange ». Mais si les laits sont mélangés dans la citerne, il n'y a plus d'inconvénients à mélanger les traites ellesmêmes, ce qui a pour avantage de rendre plus compact l'appareillage de refroidissement et d'assurer un abaissement immédiat de la température du lait fraîchement trait, à partir de la seconde traite. Les refroidisseurs à bidons ont été remplacés par les " tanks » selon l'expression anglaise, c'est-à-dire par les refroidisseurs en vrac.

Cette transformation profonde des conditions de production s'accompagne de servitudes d'ordre économique et d'ordre technologique : le refroidissement énergique du lait implique l'emploi obligatoire de refroidisseurs à machine frigorifique, ce qui n'est pas le cas pour un ramassage quotidien. L'adoption des refroidisseurs en vrac constitue une augmentation des investissements; en contrepartie les pertes de lait par acidification, notamment en saison chaude, sont pratiquement éliminées. L'utilisation de citerne permet de réduire les dépenses d'investissement et de fonctionnement de la flotte de collecte ainsi que le coût du nettoyage à l'usine. Il se trouve que, entre certaines limites, l'utilisation, onéreuse, des refroidisseurs en vrac est en grande partie compensée par les économies faites sur la collecte. En d'autres termes, l'amélioration des conditions de travail, par une meilleure organisation, n'est pas la cause d'un accroissement sensible du coût de production bien que l'investissement initial pour l'équipement de refroidissement à la production et les citernes de ramassage représente approximativement le double de l'investissement de l'usine elle-même.

Si on peut conserver le lait à la ferme de façon à ne le ramasser que tous les $2 \mathrm{j}$, pourquoi n'adopte-t-on pas une fréquence encore plus petite, du moins aux époques de faible production, de façon à augmenter la rentabilité du système. En fait, le problème est plus délicat car, si le nombre de germes dans le lait conservé entre $0^{\circ} \mathrm{C}+4^{\circ} \mathrm{C}$ reste à peu près constant, la nature de la flore évolue d'une flore 
normale, essentiellement lactique, vers une flore protéolytique. Le lait conservé trop longtemps, c'est-à-dire plus de $48 \mathrm{~h}$, n'est donc plus une matière première équivalente, notamment pour la fromagerie et la beurrerie, à un lait frais utilisé sans délai. Ce changement dans les caractéristiques technologiques du lait est indésirable ; aussi a-t-il fallu rechercher un compromis entre l'amélioration des conditions de travail du personnel et l'aptitude du lait à subir les processus industriels ; ce compromis consiste à ramasser le lait une fois au moins par période de $48 \mathrm{~h}$, c'est-à-dire encore à conserver le lait sur les lieux de production au maximum pendant quatre traites successives.

Pour certaines fabrications, notamment les fabrications fromagères les plus exigeantes, on peut réduire la perte d'aptitude du lait à la transformation industrielle en ramassant quotidiennement le lait de certains producteurs et tous les $2 \mathrm{j}$ celui des autres. Un tel procédé mixte n'est pas sans intérêt, même sur le plan économique, car on choisit pour les tournées quotidiennes les zones de production importante, situées près de l'usine ; le coût de ramassage de ces zones pèse finalement peu sur le prix de revient des opérations de collecte.

\section{雪}

Remarque : il existe d'autres procédés permettant d'alléger le travail en usine : par exemple, le lait collecté quotidiennement est amené à l'usine où il est refroidi et stocké dans de grands réservoirs isolés ; le travail du dimanche est reporté au lundi. Ce système, qui ne modifie ni la production, ni la collecte, est simple ; il implique cependant, un certain sur-équipement de l'usine et, de ce fait, peut être un peu plus coûteux.

\section{II. - LES CONDITIONS ESSENTIELLES DU REFROIDISSEMENT EN VRAC ET DU RAMASSAGE EN CITERNE}

Pour que la transformation du mode de production et de collecte du lait, du système traditionnel au système faisant intervenir le refroidissement et les citernes, ait des chances de réussir, notamment sur le plan économique, il était nécessaire que les conditions suivantes soient réalisées.

- installation d'appareils de refroidissement dans toutes les fermes des tournées entières de ramassage,

- adoption de matériels fiables et bon marché,

- aptitudes des cuves à être maintenues en bon état d'hygiène,

- contrôle précis des qualités et notamment de la qualité bactériologique des productions de chaque ferme,

- adoption d'un système précis et sûr de mesurage du lait livré,

- transfert rapide du lait, après homogénéisation, du bac du refroidisseur à la citerne de transport, 
- fonctionnement correct du refroidisseur de lait, notamment aux heures de traite, c'est-à-dire aux périodes de pointe pour le réseau de distribution électrique.

Les deux premières conditions ont été probablement les plus délicates à satisfaire au début de la transformation du mode de collecte. En effet, les responsables des laiteries avaient à décider d'un investissement important alors que les performances des refroidisseurs étaient mal connues, peu certaines et que le prix de ces équipements était élevé, essentiellement parce que la fabrication était assurée en toute petite série; en d'autres termes, l'industrie ne pouvait abaisser ses prix que si les séries devenaient suffisamment importantes et la demande ne pouvait augmenter que si les prix étaient abaissés de 25 à 30 p. 100.

Pour briser ce cercle vicieux d'ordre économique et technique, le Ministre de l'Agriculture a décidé d'accorder une aide, pouvant atteindre jusqu'à 30 p. 100 du prix du matériel, aux usines qui décident de transformer des tournées entières pourvu que les refroidisseurs achetés aient été agréés. L'agrément, prononcé par le Ministre de l'Agriculture, signifie que le refroidisseur a subi avec succès toute une série d'essais définis avec précision dans un protocole.
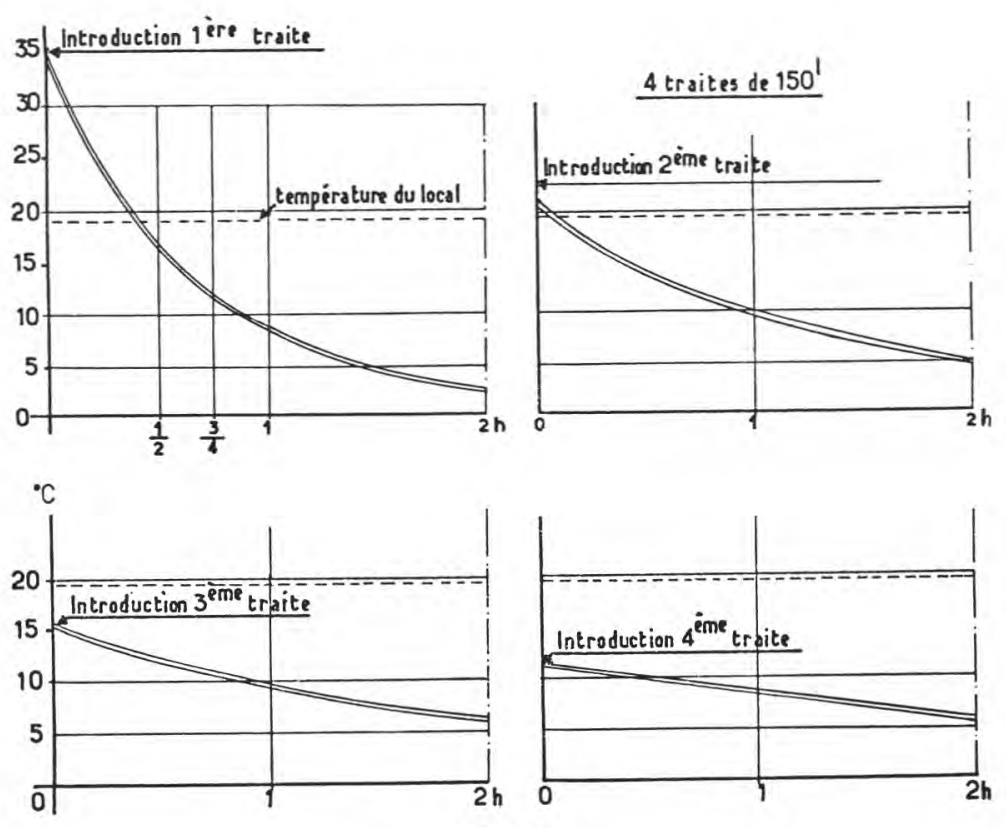

fig. 1

Refroidisseur quatre traites en détente directe Représentation de l'évolution de la température moyenne du lait en fonction du temps 
Grâce à cette politique d'incitation, la production industrielle s'est largement développée et améliorée de sorte qu'aujourd'hui les refroidisseurs agréés en France sont très appréciés à l'étranger.

\section{**}

Une autre difficulté résidait dans le choix de la capacité des refroidisseurs ; il est bien évident que tout le lait produit doit être refroidi quelle que soit l'importance des traites ; ceci implique que le volume utile de l'appareil soit égal à quatre ou deux fois la traite de pointe, selon qu'il s'agit d'un refroidisseur quatre traites ou d'un refroidisseur deux traites.

Or la production laitière étant variable au cours de l'année, on peut se rendre compte aisément que plus le coefficient d'utilisation, c'est-à-dire le rapport du volume moyen de chaque traite à celui de la traite maximale, est petit et plus lourdes sont les dépenses de refroidissement par litre de lait ; en particulier les charges d'amortissement exprimées par litre de lait, sont une fonction hyperbolique du coefficient d'utilisation. Si ce coefficient est compris entre 0,5 et 1 , les charges d'amortissement sont les plus faibles et surtout augmentent peu quand le coefficient diminue. Par contre, dès que le coefficient d'utilisation devient inférieur à 0,4 , les charges augmentent de façon très remarquée. Pour qu'un refroidisseur de lait soit exploité économiquement, il faut que le producteur régularise la lactation de son troupeau de sorte que le coefficient d'utilisation soit au moins de 0,5 alors que par le passé, si on avait refroidi le lait dans des appareils en vrac, le coefficient d'utilisation aurait été compris entre 0,2 et 0,3 . Il apparaît ainsi que les usines ont dû inciter les producteurs à mieux organiser la production laitière, ce qui n'est pas toujours aisé, eu égard notamment aux systèmes de cultures et d'exploitation des prairies.

\section{III. - L'AGREMENT DES REFROIDISSEURS}

Le Ministre de 1'Agriculture agrée uniquement les appareils ayant subi avec succès la totalité des contrôles effectués dans deux laboratoires, celui de la Station de Recherches Laitières et de Technologie de produits animaux de l'INRA à Jouy-en-Josas pour les tests de nettoyage et d'aptitude à rendre homogène le lait et le laboratoire de la Division des Industries Agricoles et Alimentaires et Froid du C.T.G.R.E.F. à Antony pour tout ce qui concerne les performances thermiques, la consommation d'énergie, et la sécurité électrique.

Sans entrer dans le détail des performances exigées, ni décrire les processus expérimentaux, il est intéressant de souligner les critères essentiels que les refroidisseurs doivent satisfaire, d'après la réglementation française du Ministère de l'Agriculture, ainsi que 
d'indiquer ce que sera vraisemblablement la norme internationale qui est actuellement à l'étude. Celle-ci est établie par un groupe de travail qui comprend les six organismes suivants :

- Comité Européen de Normalisation (C.E.N., Comité 59),

- I.S.O. (Comité 23),

- Fédération Internationale de Laiterie (F.I.L.),

- Institut International du Froid (I.I.F.),

- C.E.C.O.M.A.P. (pour les matériels frigorifiques),

- C.O.C.E.M.A. (pour les matériels de laiterie).

Ce groupe de travail est appelé « groupe CICICI » : son sigle résulte de la justaposition de la première lettre des sigles des six organisations internationales, le sigle de la Fédération Internationale de laiterie étant celui de l'expression anglaise : I.D.F.

\section{IV. - APTITUDE AU REFROIDISSEMENT}

Pour un refroidisseur quatre traites, chaque traite, introduite en une fois et représentant le quart du volume utile de la cuve, doit être refroidie de $35^{\circ} \mathrm{C}$ à $5^{\circ} \mathrm{C}$ en moins de $2 \mathrm{~h}$, dans un local dont la température est comprise entre $20^{\circ} \mathrm{C}$ et $5^{\circ} \mathrm{C}$ ou entre $32^{\circ} \mathrm{C}$ et $5^{\circ} \mathrm{C}$.

Le contrôle consiste donc à vérifier que le temps effectif de refroidissement de chacune des traites est satisfaisant quand la température ambiante est de $20^{\circ} \mathrm{C}$ ou de $32^{\circ} \mathrm{C}$ et quand elle est de $5^{\circ} \mathrm{C}$.

Pour un refroidisseur deux ou quatre traites, le contrôle de l'aptitude au refroidissement est complété par la vérification du refroidissement de $35^{\circ} \mathrm{C}$ à $5^{\circ} \mathrm{C}$, de deux traites, représentant chacune la moitié du volume, est obtenu en moins de $3 \mathrm{~h}$, dans les mêmes conditions d'ambiance.

$$
\text { ** }
$$

Il convient de souligner l'importance des essais effectués en local froid $\left(+5^{\circ} \mathrm{C}\right)$. Bien que cela ne soit pas évident, les performances de refroidissement par basse température ambiante peuvent être médiocres, selon le procédé adopté pour la détente du fluide frigorigène. En outre, le fonctionnement du dispositif automatique règlant la température du lait peut être perturbé si les caractéristiques du milieu extérieur subissent des fluctuations de grande amplitude. Dans la pratique, les refroidisseurs de lait sont soumis, entre l'été et l'hiver, à des ambiances dont la température varie de plus de $20^{\circ} \mathrm{C}$. Cette vérification, importante, n'était effectuée qu'en France ; elle est maintenant retenue notamment dans le projet de norme internationale ainsi que dans les recommandations de la Fédération Internationale de Laiterie et du C.E.C.O.M.A.F. 
Il est bien clair que l'aptitude au refroidissement doit être indépendante du mode de fonctionnement du système frigorifique. Il existe deux grandes catégories d'appareils selon le mode de transfert de chaleur :

- les refroidisseurs en détente directe, dans lesquels l'évaporation du fluide frigorigène est effectuée dans un échangeur en contact avec la cuve à lait (ou avec le lait); la puissance frigorifique de la machine équipant ces appareils doit être suffisamment grande pour fournir tout le froid nécessaire pendant la durée du refroidissement,

- les refroidisseurs à accumulation de glace. Le refroidissement est obtenu par ruissellement d'eau glacée à l'extérieur et à partir du bord supérieur de la cuve à lait. L'eau glacée circule en circuit fermé entre la cuve à lait et l'évaporateur de la machine frigorifique sur lequel est accumulée, pendant les heures précédant la traite, une certaine quantité de glace ; celle-ci constitue un volant de froid.

Les refroidisseurs en détente directe assure le refroidissement en un temps à peu près indépendant des conditions de remplissage de la cuve; en d'autres termes, si le lait est introduit dans l'appareil progressivement, pendant toute la durée de la traite, la durée du refroidissement est semblable à celle observée quand tout le lait, à la même température, est transvasé en une seule fois. Pour les refroidisseurs à accumulation de glace, la durée totale du refroidissement augmente d'autant plus que le remplissage de l'appareil dure davantage. Par exemple, si un refroidisseur de lait refroidit une traite introduite en une seule fois en $2 \mathrm{~h}$, il refroidit la même quantité de lait en un temps voisin de $3 \mathrm{~h}$, si le transvasement a été effectué en 1 h 30 .

Il apparaît que les performances pratiques des refroidisseurs de lait en détente directe peuvent être correctement appréciés par une mesure faite dans des conditions conventionnelles, avec introduction en une fois du volume correspondant à une traite. Pour les refroidisseurs à accumulation de glace, par contre, les résultats de mesure conventionnelle, avec introduction en une fois, ne reproduisent pas les performances correspondant à l'usage habituel.

Pour assurer le refroidissement du lait dans les mêmes conditions pratiques, on pourrait imposer un temps de refroidissement conventionnel plus court pour les appareils à accumulation de glace que pour ceux à détente directe. La différence entre ces deux temps devrait être comprise entre 30 et $60 \mathrm{mn}$. Une telle spécification serait illogique puisqu'elle conduirait à exprimer les performances d'appareils en pratique équivalents par des valeurs apparemment meilleures pour l'un des deux.

Pour lever cette difficulté, le groupe international de normalisation des refroidisseurs de lait, sur proposition de son représentant français, a adopté une définition plus complète du refroidissement. Un refroidisseur doit assurer le refroidissement de chaque traite 
introduite en une fois de $35^{\circ} \mathrm{C}$ à $4^{\circ} \mathrm{C}$ (le groupe international a fixé la température limite à $4^{\circ} \mathrm{C}$ et non à $5^{\circ} \mathrm{C}$, comme dans le protocole français) en un temps total n'excédant pas $3 \mathrm{~h}$ pour tous les appareils, deux et quatre traites, et le refroidissement de la seconde traite de $10^{\circ} \mathrm{C}$ à $4^{\circ} \mathrm{C}$ en un temps n'excédant pas $1 \mathrm{~h} 30$. Les temps doivent être obtenus dans une ambiance à $32^{\circ} \mathrm{C}$ et à $5^{\circ} \mathrm{C}$.

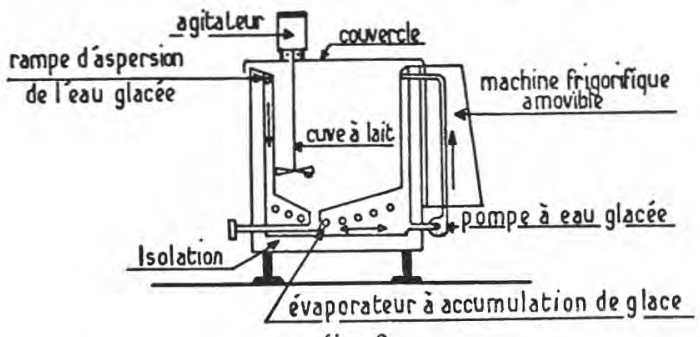

fig. 2

Schéma de principe d'un refroidisseur « à accumulation de glace »

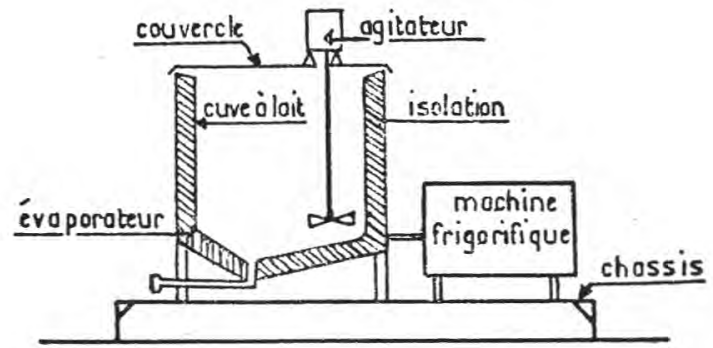

fig. 3

Schéma de principe d'un refroidisseur (en détente directe) et à évaporateur extérieur

Pour les refroidisseurs en détente directe, la spécification la plus dure est celle du temps total de refroidissement. Par contre, pour ceux à accumulation de glace, la spécification la plus sévère est celle du refroidissement de $10^{\circ} \mathrm{C}$ à $4^{\circ} \mathrm{C}$ de la seconde traite ; si cette condition est satisfaite, le temps total est alors nettement inférieur à $3 \mathrm{~h}$; il est voisin de $2 \mathrm{~h} 30$, l'introduction d'une traite étant faite en une fois.

Dans la définition de la performance de refroidissement, la quantité de lait, introduite à chaque traite par période égale de $12 \mathrm{~h}$, est de 25 ou 50 p. 100 du volume utile de la cuve, selon qu'il s'agit d'appareils quatre ou deux traites. Dans la pratique, le temps séparant la traite du matin et la traite du soir est de l'ordre de 8 à $10 \mathrm{~h}$, de celui de la traite du soir à celle du matin, de l'ordre de 14 à 16 h. Pour une production quotidienne déterminée, la traite du matin est à peu près les $6 / 10$ de ce volume et la traite du soir à peu près les $4 / 10$. On pourrait définir les performances des refroidis- 
seurs de lait en ne tenant compte de ces inégalités de volume et de temps comme cela est le cas pour certaines normes étrangères. En fait, il est apparu qu'un refroidisseur apte à refroidir toutes les $12 \mathrm{~h}$ des traites égales est également capable de refroidir correctement les traites du matin et du soir. Il n'est donc pas nécessaire de compliquer le processus expérimental.

\subsection{Aptitude à la conservation}

Les refroidisseurs de lait doivent être capables de maintenir en permanence le lait entre $0^{\circ} \mathrm{C}$ et $4^{\circ} \mathrm{C}$, jusqu'au moment du transvasement dans la citerne de transport; ceci implique que la cuve soit suffisamment isolée thermiquement et que, éventuellement, les systèmes de production de froid et d'agitation soient mis en service pendant la période séparant deux traites.

L'isolation est définie, dans le protocole français, par le coefficient global de transfert de chaleur (coefficient $\mathrm{K}$ ), qui doit être inférieur à $1.2 \mathrm{~W} / \mathrm{m}^{2}{ }^{\circ} \mathrm{C}$ ou $1 \mathrm{kcal} / \mathrm{h} \cdot \mathrm{m}^{2}{ }^{\circ} \mathrm{C}$. Ce coefficient, mesuré dans des conditions expérimentales bien définies. correspond à un concept habituel pour les frigoristes, mais constitue une information peu évocatrice pour les laitiers.

Ces derniers sont plus sensibles à la définition de l'isolation par l'élévation de température observée pendant un laps de temps déterminé, la machine étant à l'arrêt et le local étant à température constante et définie. Généralement, on admet que le réchauffement moyen ne doit pas dépasser $3^{\circ} \mathrm{C}$ par période de $12 \mathrm{~h}$, quand la cuve est complètement remplie, pour une ambiance extérieure maintenue constante à $+32^{\circ} \mathrm{C}$.

De fait, les deux spécifications, coefficient $\mathrm{K}$ et réchauffement du lait, qui correspondent au même phénomène de transfert de chaleur à travers les parois de la cuve, ne sont pas équivalents ; pour un appareil de petit volume, c'est-à-dire de grande surface extérieure par litre de lait logé, il est plus aisé de satisfaire le critère du coefficient $\mathrm{K}$ que celui de l'élévation de la température du lait. La conclusion est inverse pour les appareils de grand volume représentant une plus faible surface extérieure par litre logé.

La réglementation française a adopté la notion du coefficient $\mathrm{K}$. Le projet de réglementation internationale a préféré le concept de l'élévation de la température du lait; il est cependant possible que les deux concepts soient retenus et définis par une valeur limite imposée.

\subsection{Consommation électrique et sécurité}

La réglementation française exige que le compte rendu du laboratoire mentionne la consommation électrique mesurée dans des conditions définies de température ambiante et de remplissage de la cuve ; cependant, aucune valeur limite n'est imposée. Il est admis, en 
effet, que les utilisateurs doivent apprécier eux-mêmes le poids de la dépense d'énergie électrique sur le prix global du refroidissement du lait.

Dans la pratique, il existe une différence notable entre les deux types de refroidisseurs de lait.

Les refroidisseurs en détente directe consomment à peu près deux fois moins d'énergie électrique que ceux à accumulation de glace, dans des conditions similaires de service. Par contre, les premiers nommés exigent une puissance électrique installée de deux à quatre fois supérieure à celle nécessaire pour les appareils à accumulation de glace.

\section{**a.}

Les règles de sécurité électrique doivent être bien entendu impérativement respectées. Il est prévu pour la réglementation française la vérification de cette qualité. Cette question n'a pas encore été discutée sur le plan international, pour le moment du moins.

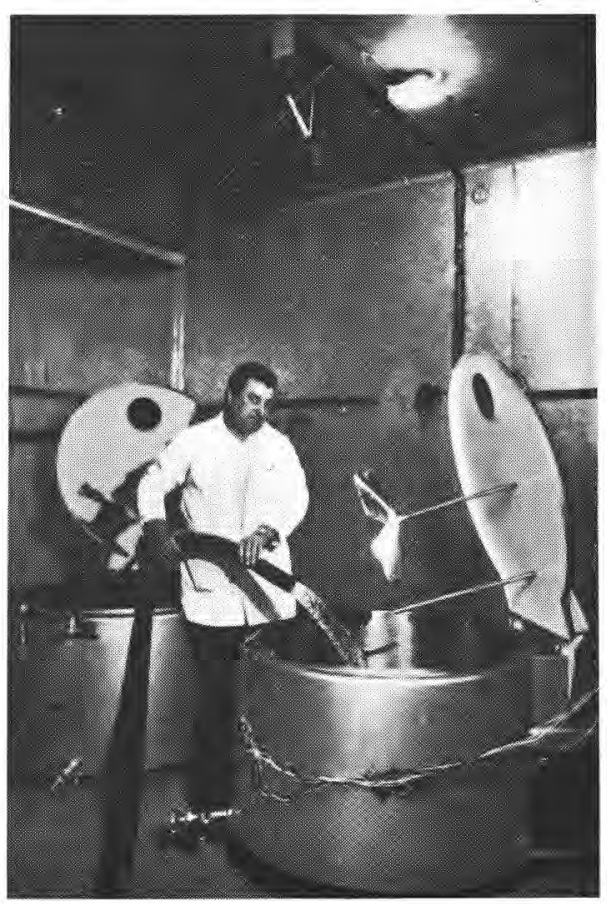

fig. 4

Un appareil en cours de remplissage pour des essais thermiques ; à noter que la détermination de performances thermiques est faite avec de l'eau 


\subsection{Apitude à maintenir une bonne hygiène}

L'aptitude à l'hygiène d'un refroidissement de lait dépend des caractéristiques de construction de la cuve et des conditions de nettoyage de celle-ci.

Les caractéristiques de construction sont définies par la nature de la cuve, en acier inoxydable 18/08 finement poli, et la façon de réaliser les angles et jonctions.

La réglementation nationale prévoit la vérification de l'aptitude au nettoyage par des essais qui consistent à réaliser de façon conventionnelle et accélérée un certain état de souillure, à procéder au nettoyage selon les directives du constructeur et à déterminer l'état de pollution résiduelle des surfaces nettoyées.

Sur le plan international, on s'est orienté vers une description détaillée de la construction des refroidisseurs de lait; il ne semble pas que des essais conventionnels d'aptitude au nettoyage seront repris dans le texte international.

Dans le texte français et le projet international, les conditions de nettoyage des appareils sont complétées par un certain nombre de directives concernant l'évacuation des eaux de lavage, le nettoyage de toutes les parties mobiles ou inmmergées dans le lait, telles que agitateur, sonde thermométrique, etc.

Le maintien d'une bonne hygiène ne semble plus être une difficulté, du moins pour les refroidisseurs actuels à nettoyage manuel ; par contre, avec le développement prévisible des refroidisseurs de grand volume, à nettoyage automatique, la définition de l'aptitude à maintenir une bonne hygiène et la vérification de cette aptitude risquent de constituer des problèmes assez délicats.

\subsection{Aptitudes à homogénéiser le lait}

Normalement, le lait est agité pendant la période de refroidissement. Il peut être agité à intervalles de temps réguliers pendant la phase de stockage pour maintenir l'ensemble du lait à température homogène et ainsi éliminer l'apparition progressive de « points chauds ", notamment au niveau de la sonde de vidange.

Une agitation trop fréquente du lait est jugée de façon peu favorable par les spécialistes du lait ; elle risque d'avoir une action sur la lipolyse.

Cependant, on tend à considérer qu'il n'y a pas d'inconvénient à ce que le lait soit homogénéisé de façon douce et peu fréquente. Enfin, le lait doit être rendu homogène en un temps court au moment d'un transvasement dans la citerne de ramassage. Les appareils sont normalement munis d'un système de commande manuelle de l'agitateur qui est utilisé au moment de la vidange. 
L'agitation du lait est, d'après la réglementation française, suffisante si la distribution de la matière grasse est homogène en $5 \mathrm{mn}$. En fait, ce temps est trop long et en pratique les appareils sont aptes à homogénéiser le lait en $2 \mathrm{mn}$. Cette dernière valeur est d'ailleurs celle retenue dans le projet international.

Le dessin des pales de l'agitateur doit être tel que le lait soit lentement brassé et ne soit ni barraté, ni émulsionné. Cette condition conduit à l'adoption d'hélice de grand diamètre tournant entre 30 et $50 \mathrm{t} / \mathrm{mn}$ et à bannir absolument les petits agitateurs bon marché, à $1500 \mathrm{t} / \mathrm{mn}$, qui avait été proposés dans les appareils plus anciens.

\subsection{Autres caractéristiques}

Il serait également possible de soumettre un refroidisseur de lait à d'autres contrôles, tels que la vérification de sa fiabilité, la vérification de la précision de mesurage du volume de lait, etc.

En fait, pour les professionnels de la production et de la construction, les spécialisations rappelées ci-dessus sont les plus importantes. Les textes réglementaires nationaux et internationaux ont limité la liste des spécifications à vérifier à celles indiquées ci-dessus.

\section{V. - DEFINITION D'UN REFROIDISSEUR DE LAIT}

Comme pour beaucoup d'appareils fabriqués en série, il devrait être suffisant de donner l'appellation commerciale d'un appareil pour que celui-ci soit complètement défini.

En fait, le problème des refroidisseurs de lait est plus délicat ; ces appareils sont le résultat de l'assemblage de sous-ensembles four-

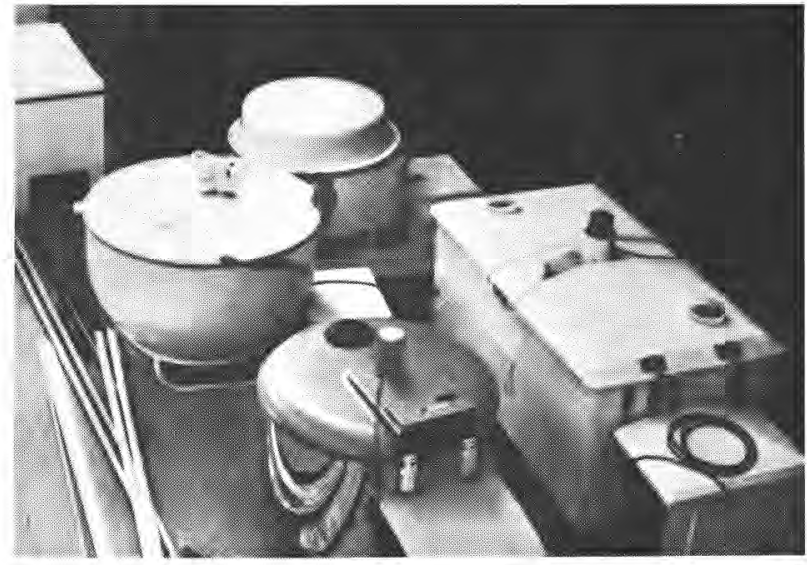

fig. 5

La disposition des sondes thermométriques dans la cuve à lait pour les essais thermiques 
nis par différentes industries : industrie du matériel de laiterie pour la cuve, constructeurs de machines frigorifiques pour le groupe, fabricants d'automates pour le système automatique.

Un même assembleur peut se procurer auprès de différents fabricants des éléments qui peuvent ne pas être équivalents : par exemple, des groupes frigorifiques de différentes marques, ayant la même puissance, n'assurent pas nécessairement les mêmes performances lorsqu'ils sont montés sur une même cuve.

C'est la raison pour laquelle dans l'agrément du Ministère de l'Agriculture, un refroidisseur est défini non seulement par sa marque commerciale et le nom de l'assembleur, mais par un certain nombre d'informations supplémentaires : appareil 2 et 4 traites, aptitude à assurer le refroidissement en atmosphère à $+20^{\circ} \mathrm{C}$ ou à $+32^{\circ} \mathrm{C}$, appellation du groupe frigorifique, marque de l'agitateur, marque du thermostat.

\section{VI. - CONCLUSION}

L'évolution des conditions de production du lait, notable depuis ces dernières années, n'est probablement pas achevée.

On peut penser que les nombreux petits troupeaux importants, que la production de lait de qualité, notamment de qualité bactériologique satisfaisante, continuera à se généraliser, que les producteurs continueront à chercher une meilleure organisation, par exemple par la construction des fermes laitières coopératives, de façon à alléger et à améliorer les conditions de travail, etc.

Dans une dizaine d'années, on ressentira peut-être la même impression de "périmé ", à propos des refroidisseurs actuels que celle que l'on éprouve aujourd'hui quand on évoque les procédés préconisés avant 1965 pour le refroidissement du lait à la ferme. 